\title{
FTO effect on energy demand versus food intake
}

\author{
Arising from: J. Fischer et al. Nature 458, 894-898 (2009)
}

An intronic single nucleotide polymorphism (SNP) (rs9939609) close to the fat mass and obesity associated gene (FTO) was the first SNP to be discovered with common variants linked to body mass index ${ }^{1}$; at least seven studies in humans have implicated this SNP with variations in food intake and satiety ${ }^{2-8}$, and four studies have rejected an effect on energy expenditure normalized for body weight ${ }^{2,5,6,8}$. Fischer et al. ${ }^{9}$ recently constructed a mouse in which the homologous Fto gene was inactivated $\left(\mathrm{Fto}^{-{ }^{-}}\right)$and showed that these mice were protected from obesity. This observation strongly implicates the effects of the intronic SNP rs9939609 as arising due to an effect on the closest gene (FTO). However, the suggested mechanism underlying this effect in mice was opposite to that in humans. The Fto ${ }^{-1-}$ mice showed no significant differences in food intake relative to wild-types litter-mates ${ }^{9}$ but had an elevated metabolic rate. The apparent contrasting effects of the gene in humans and mice is worthy of closer investigation.

The difference in body fatness between $\mathrm{Fto}^{-1-}$ and $\mathrm{Fto}^{+/ \text {? }}$ mice was about $3.5 \mathrm{~g}$ in males and $1 \mathrm{~g}$ in females at 20 weeks old (Fig. 3 in ref. 9). One gram of fat is equal to about $39 \mathrm{~kJ}$ of energy. Assuming the mice weaned at 3 weeks of age, then over 17 weeks ( 120 days) the energy imbalance necessary to generate this difference is about $0.33 \mathrm{~kJ}^{-1}{ }^{-1}$ for females and about $1.16 \mathrm{~kJ} \mathrm{day}^{-1}$ for males. The energy density of standard rodent chow is about $17 \mathrm{~kJ} \mathrm{~g}^{-1}$. Hence the difference in food intake between genotypes required to generate the observed differences in body fatness was about $0.02 \mathrm{~g} \mathrm{day}^{-1}$ in the females and $0.07 \mathrm{~g} \mathrm{day}^{-1}$ in males. Food intake in rodents is potentially much easier to study than in humans because food intake in rodents can be measured accurately, they can be fed from a single food source removing the influence of differences in macronutrient composition and they can be monitored for much longer periods-exceptionally over their entire lives. Nevertheless, given that individual variability in food intake in mice has a standard deviation of about $0.3 \mathrm{~g} \mathrm{day}^{-1}$ then the required sample size to detect these effect sizes, using a 3 level one-way ANOVA (as analysed in ref. 9) with a power of $80 \%$ and $\alpha=0.05$ is 355 per group for males, and 4,337 for females. Fischer et al. ${ }^{9}$ only presented data for food intake in female mice. They used a total sample of 31 female animals. The post hoc estimated power to detect the effect size of $0.02 \mathrm{~g} \mathrm{day}^{-1}$ in food intake was only $5.1 \%$. Many studies of the effects of genes on food intake are underpowered and this critique could have been levelled at any number of recent studies. An example of a correctly powered study is that of ref. 10 on the effects of insulin receptor substrate-1 (Irs1) null mice. In that study using a sample of 12 null and 12 wild-type mice it was shown that the effect of disruption of Irs1 on longevity did not come about because of an effect of the inactivation of Irs1 on food intake. Although the sample size in this latter study may not seem very different from that used by Fischer et al. ${ }^{9}$, the key difference between the studies is the magnitude of the effect size that is being detected. In ref. 10 the effect size is $50 \times$ greater than that being detected by Fischer et al. ${ }^{9}$, consequently the power to detect or reject this effect was $98 \%$. Sample sizes in experiments like those conducted in ref. 9 need to be sufficient to detect the effect size that generates the observed difference in fatness. Clearly this experiment was underpowered and the rejection of an effect of the Fto genotype on food intake has a strong likelihood of being a type II error.
The second potential issue with the findings of Fischer et al. ${ }^{9}$ relates to the estimated energy expenditure. First, there is the same power issue highlighted above with respect to food intake, except that there is even less power in the expenditure measures as the total sample is only 23 individuals. More importantly, to evaluate the role of expenditure differences Fischer et al. (Fig. 4 in ref. 9) made a simple division of the metabolism by the lean body mass (LBM). This is a common practice to attempt to normalize for body size effects. However, simple division by LBM can generate a spurious elevation of metabolic rate if the intercept of the relationship between metabolism and LBM is not zero ${ }^{11}$, as is often the case. The suggested increased metabolism in the Fto ${ }^{-1-}$ animals is potentially an artefact of using this analysis method. The metabolic rates of these animals may only seem higher because the expenditure has been divided by a smaller lean body mass. If these data had been analysed using ANCOVA $^{11}$ the effect of genotype would very probably disappear (but then the absence of an effect could also be a type II error because of the power issue).

Overall the construction of the Fto ${ }^{-1-}$ mouse is a great achievement that identifies the FTO gene as the prime candidate being affected by the intronic rs9939609 SNP. Given the roles of many genes on both intake and expenditure it is entirely possible that the mechanisms by which Fto influences energy balance in the mouse, as claimed by Fischer et al. ${ }^{9}$, really do contrast the effects in humans ${ }^{2-8}$. Unfortunately the issue of lack of power and the complexity of normalization of energy expenditure measurements mean that at present it is impossible to judge.

\section{John R. Speakman}

${ }^{1}$ Institute of Biological and Environmental Sciences, University of Aberdeen, Aberdeen AB24 2TZ, Scotland, UK.

e-mail: j.speakman@abdn.ac.uk

Received 27 November; accepted 1 December 2009.

1. Frayling, T. M. et al. A common variant in the FTO gene is associated with body mass index and predisposes to childhood and adult obesity. Science 316, 889-894 (2007).

2. Speakman, J. R., Rance, K. A. \& Johnstone, A. M. Polymorphisms of the FTO gene are associated with variation in energy intake, but not energy expenditure. Obesity (Silver Spring) 16, 1961-1965 (2008)

3. Wardle, J. et al. Obesity associated genetic variation in FTO is associated with diminished satiety. J. Clin. Endocrinol. Metab. 93, 3640-3643 (2008).

4. Timpson, N. J. et al. The fat mass- and obesity-associated locus and dietary intake in children. Am. J. Clin. Nutr. 88, 971-978 (2008).

5. Do, R. et al. Genetic variants of FTO influence adiposity, insulin sensitivity, leptin levels, and resting metabolic rate in the Quebec Family Study. Diabetes 57, 1147-1150 (2008).

6. Haupt, A. et al. Variation in the FTO gene influences food intake but not energy expenditure. Exp. Clin. Endocrinol. Diabetes 117, 194-197 (2009).

7. Wardle, J. et al. The FTO gene and measured food intake in children. Int. J. Obes. 33, 42-45 (2009)

8. Cecil, J. E. et al. An obesity associated FTO gene variant and increased energy intake in children. N. Engl. J. Med. 359, 2558-2566 (2008).

9. Fischer, J. et al. Inactivation of the Fto gene protects against obesity. Nature 458, 894-898 (2009).

10. Selman, C. et al. Evidence for lifespan extension and delayed age-related biomarkers in insulin receptor substrate 1 null mice. FASEB J. 22, 807-818 (2008).

11. Arch, J. R. S. et al. Some mathematical and technical issues in the measurement and interpretation of open-circuit indirect calorimetry in small animals. Int. J. Obes. 30, 1322-1331 (2006).

Competing financial interests: declared none.

doi:10.1038/nature08807 


\section{Fischer et al. reply}

Replying to: J. R. Speakman Nature 464, doi:10.1038/nature08807 (2010)

The human studies on FTO reported an association of an intronic single nucleotide polymorphism (SNP) with obesity. Our report of mice with the targeted inactivation of the Fto gene demonstrated a direct role of Fto in energy homeostasis ${ }^{1}$. We have shown that the absence of Fto protein results in leanness and that Fto deficiency affects energy homeostasis. Speakman ${ }^{2}$ exemplifies that the experiments performed in mice ${ }^{1}$ conflict with results in humans carrying the FTO risk allele presenting hyperphagia and increased caloric intake ${ }^{3-9}$

The majority of studies examining the effects of FTO risk SNPs and determinants of adiposity in humans have reported association with food intake and/or appetite, and none has reported a significant effect on energy expenditure. However, the effects are modest in size and the precise measurement of the components of energy balance in human is fraught with difficulty, so it would therefore be premature to state conclusively that FTO affects human adiposity only through an effect on food intake.

With respect to the analysis of energy expenditure in our mouse model, correction of energy expenditure, that is, $\mathrm{O}_{2}$ consumption to lean body mass, represents at this point a standard procedure in mouse phenotyping. This kind of data analysis was specifically requested by the reviewers of our manuscript. Another group has since reported the generation and analysis of a mouse model carrying a point mutation in the murine Fto gene as a consequence of $N$-ethyl$\mathrm{N}$-nitrosourea mutagenesis ${ }^{10}$. The phenotype of this mouse line closely resembles the knockout phenotype ${ }^{1}$, although the alterations in overall body size are much more moderate.

However, this model also presents reduced fat mass and resistance to high-fat-induced obesity. Notably, in line with our report, even in the absence of major weight differences and with unaltered lean mass, mice carrying the Fto point mutation show increased energy expenditure as reported for the knockout model ${ }^{10}$.

Taken together, our report on Fto-deficient mice provides a direct study on the role of the Fto protein in the absence of dysregulated Ftm (also known as Rpgrip1l) expression, the key findings of which have already been reproduced in an independent mouse model with altered Fto function. The observed reduction in fat mass does represent an important step towards the further understanding of FTO biology and we are confident that this and other mouse models will provide important additional insights into the function of FTO in the near future.

Julia Fischer ${ }^{1}$, Linda Koch ${ }^{2,3}$, Christian Emmerling ${ }^{1}$, Jeanette Vierkotten ${ }^{1} \dagger$, Thomas Peters ${ }^{1}+$, Jens C. Brüning ${ }^{2,3,4}$ \& Ulrich Rüther ${ }^{1}$

${ }^{1}$ Institute for Animal Developmental and Molecular Biology, Heinrich Heine University, Universitätsstr. 1, D-40225 Düsseldorf, Germany. email: ruether@uni-duesseldorf.de

${ }^{2}$ Department of Mouse Genetics and Metabolism, Institute for Genetics and Second Department of Internal Medicine, University of Cologne,

D-50674 Cologne, Germany.

${ }^{3}$ Center of Molecular Medicine Cologne (CMMC) and Cologne

Excellence Cluster on Cellular Stress Responses in Aging-Associated

Diseases (CECAD), University of Cologne, D-50674 Cologne, Germany.

${ }^{4}$ Max-Planck Institute for the Biology of Ageing, D-50674 Cologne,

Germany.

$\uparrow$ Present addresses: Department of Dermatology, Center of Molecular Medicine Cologne (CMMC), University of Cologne, D-50674 Cologne, Germany (J.V.); Epidauros Biotechnologie AG, D-82347 Bernried, Germany (T.P.).

1. Fischer, J. et al. Inactivation of the Fto gene protects against obesity. Nature 458, 894-898 (2009).

2. Speakman, J. R. FTO effect on energy demand versus food intake. Nature 464, doi:10.1038/nature08808.

3. Speakman, J. R., Rance, K. A. \& Johnstone, A. M. Polymorphisms of the FTO gene are associated with variation in energy intake, but not energy expenditure. Obesity (Silver Spring) 16, 1961-1965 (2008).

4. Wardle, J. et al. Obesity associated genetic variation in FTO is associated with diminished satiety. J. Clin. Endocrinol. Metab. 93, 3640-3643 (2008).

5. Timpson, N. J. et al. The fat mass- and obesity-associated locus and dietary intake in children. Am. J. Clin. Nutr. 88, 971-978 (2008).

6. Do, R. et al. Genetic variants of FTO influence adiposity, insulin sensitivity, leptin levels and resting metabolic rate in the Quebec Family Study. Diabetes 57, 1147-1150 (2008).

7. Haupt, A. et al. Variation in the FTO gene influences food intake but not energy expenditure. Exp. Clin. Endocrinol. Diabetes 117, 194-197 (2009).

8. Wardle, J. et al. The FTO gene and measured food intake in children. Int. J. Obes. 33, 42-45 (2009)

9. Cecil, J. E. et al. An obesity associated FTO gene variant and increased energy intake in children. N. Engl. J. Med. 359, 2558-2566 (2008).

10. Church, C. et al. A mouse model for the metabolic effects of the human fat mass and obesity associated FTO gene. PLoS Genet. 5, e1000599 (2009).

Competing financial interests: declared none

doi:10.1038/nature08808 